\title{
FOREWORD
}

\section{Special Section on Recent Progress in Electromagnetic Theory and Its Application}

The purpose of this special section is to present a collection of original papers that give an overview of recent progress of research and development in electromagnetic theory and its applications, including those related papers presented at the 2016 Symposium on Electromagnetic Theory, held in Wakayama, Japan on November 17-19, 2016. Presentations in the International Symposia and Meetings held during 2016, which include AP-S/URSI2016, PIERS2016, ICEAA2016 and ISAP2016, were also considered to be the parts of this special section. In response to the call-for-papers, we received some potential paper manuscripts. After careful review, 4 papers and 5 brief papers, were accepted in this special section.

The topics of accepted papers include various topics such as electromagnetic scattering theory, a new approach in scattering analysis and inverse problem and their applications. We hope that the readers find this special section useful in the research of the electromagnetic theory and its applications. We would like to express our sincere appreciations to all the authors of contributed papers for their efforts in preparing the manuscripts and to all the reviewers for their adequate judgments and valuable comments.

We are also indebted to the editorial committee members for their dedicated efforts in organizing this special section. Especially, we would like to express our sincere thanks to Yasuhiro Nishioka of Mitsubishi Electric Corp. and Professor Takuya Sakamoto of University of Hyogo, who played important roles in the publication of this special section.

Editorial Committee of the Special Section on Recent Progress in Electromagnetic Theory and Its Application:

Secretaries:

Yasuhiro Nishioka (Mitsubishi Electric Corp.)

Takuya Sakamoto (University of Hyogo)

Associate Editors:

Yoshiaki Ando (The Univ. of Electro-Communications)

Yoshio Inasawa (Mitsubishi Electric Corp.)

Tetsuya Ueda (Kyoto Inst. of Tech.)

Shinichiro Ohnuki (Nihon Univ.)

Ryosuke Ozaki (Nihon Univ.)

Keiji Goto (Nat'l Defense Academy of Japan)

Ryoichi Sato (Niigata Univ.)

Toshihiko Shibazaki (Tokyo Metro. College of Indust. Tech.)

Yukihisa Suzuki (Tokyo Metro. Univ.)

Kazunori Takahashi (OYO Corp.)

Masahiro Tanaka (Gifu Univ.)

Norimasa Nakashima (Fukuoka Inst. of Tech.)

Takuichi Hirano (Tokyo Inst. of Tech.)

Koichi Hirayama (Kitami Inst. of Tech.)

Motoyuki Sato and Akira Hirose, Guest Editors-in-Chief 
Motoyuki Sato (Senior Member)

received the B.E., M.E degrees, and Dr. Eng. degree in information engineering from the Tohoku University, Sendai, Japan, in 1980, 1982 and 1985, respectively. Since 1997 he is a professor at Tohoku University and a distinguished professor of Tohoku University between 2007-2011, and he was the Director of Center for Northeast Asian Studies, Tohoku University during 2009-2013. From 1988 to 1989, he was a visiting researcher at the Federal German Institute for Geoscience and Natural Resources (BGR) in Hannover, Germany. His current interests include transient electromagnetics and antennas, radar polarimetry, ground penetrating radar (GPR), borehole radar, electromagnetic induction sensing, interferometric and polarimetric SAR. He has conducted the development of GPR sensors for humanitarian demining, and his sensor ALIS which is a hand-held dual sensor, has detected more than 80 mines in mine fields in Cambodia since May 2009. He is a visiting Professor at Jilin University, China, Delft University of Technology, The Netherlands, and Mongolian University of Science and Technology. He was the Chair of the Technical Committee on Electromagnetic Theory in Electronics Society of the IEICE in 2015-2016. He was the technical chair of GPR96 and the general chairperson of IGARSS2011. Dr. Sato is a Fellow of IEEE.

Akira Hirose (Senior Member) received the Ph.D. degree in electronic engineering from the University of Tokyo in 1991. He is presently Professor with Department of Electrical Engineering and Information Systems, the University of Tokyo. The main fields of his research interests include wireless electronics and neural networks. He served as the Founding President of APNNS (2016), President of the JNNS (2013-2015), Vice President of the IEICE Electronics Society (2013-2015), Editor-in-Chief of the IEICE Transactions on Electronics (2011-2012), Associate Editor of journals such as the IEEE Transactions on Neural Networks (2009-2011), IEEE Geoscience and Remote Sensing Newsletter (2009-2012), Chair of the Neurocomputing Technical Group in the IEICE, General Chair of International Conference on Neural Information Processing (ICONIP) 2016 Kyoto, and General Chair of the 2013 AsiaPacific Conference on Synthetic Aperture Radar (APSAR 2013) in Tsukuba. He currently serves as the General Chair of International Geoscience and Remote Sensing Symposium (IGARSS) 2019 Yokohama. Dr. Hirose is a Fellow of the IEEE.
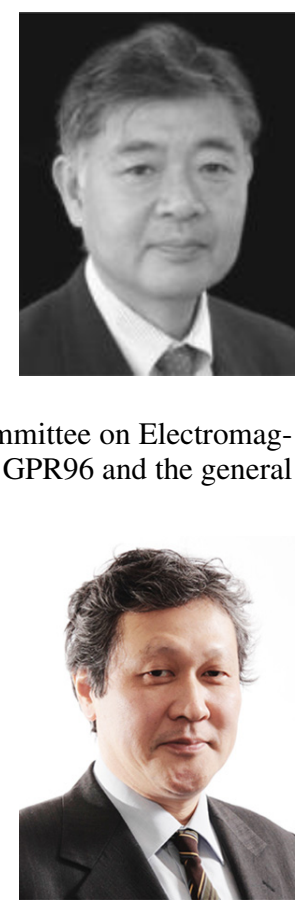